\title{
Germanium hut nanostressors on freestanding thin silicon membranes
}

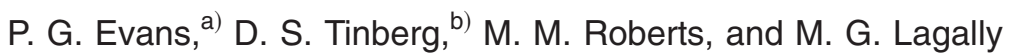 \\ Department of Materials Science and Engineering and Materials Science Program, University of Wisconsin, \\ Madison, Wisconsin 53706
}

Y. Xiao, B. Lai, and Z. Cai

Advanced Photon Source, Argonne National Laboratory, Argonne, Illinois 60439

(Received 25 March 2005; accepted 29 June 2005; published online 11 August 2005)

\begin{abstract}
The heteroepitaxial growth of Ge on thin Si membranes can lead to significant bending under self-assembled Ge hut nanostructures. Undercut silicon-on-insulator mesas approximate a $\mathrm{Si}$ freestanding membrane and serve as a crystalline substrate for the growth of Ge huts. Synchrotron x-ray microdiffraction shows a local curvature on the lateral scale of the size of the hut and an overall bending of the freestanding region. In comparison with conventional mechanically rigid substrates, the freestanding film can bend significantly. We have found a local radius of curvature of $6 \mu \mathrm{m}$ beneath huts on 30-nm-thick Si membranes. (C) 2005 American Institute of Physics. [DOI: $10.1063 / 1.2031941]$
\end{abstract}

The development of thin mechanically isolated crystalline layers, particularly in silicon-on-insulator (SOI) structures, has led to a new range of phenomena in strained thin films. The thickness of the Si template layer in SOI can be reduced to the point that, when the template layer is released from the supporting handle wafer, the strain arising from the epitaxial mismatch between the thin substrate and a deposited thin film is partitioned elastically between them. Lattice strains of more than $1 \%$ have been produced in SOI template layers in this manner. ${ }^{1}$ Decreasing the template layer thickness also blurs the distinction between quantum-dot-scale stressors, which are typically thought to produce purely local distortions, ${ }^{2}$ and blanket films that produce large-scale wafer curvature. $^{3}$ The mechanical response of conventional substrates, with thicknesses of hundreds of microns, is a powerful tool for understanding stress evolution in thin-film growth averaged over macroscopic lateral lengths. ${ }^{4-6}$ Thin membranes have the potential to be similarly sensitive probes for nanometer-scale mechanics.

Ge hut clusters are self-organized structures with typical dimensions of tens of nanometers that form when a Ge film thicker than the planar three-monolayer (ML) wetting layer is deposited on $\mathrm{Si}(001){ }^{7}$ These clusters are the response of the Ge film to the large epitaxial mismatch between $\mathrm{Si}$ and Ge. The lattice mismatch between Si and Ge also leads, however, to large stresses on the Si substrate. We have found that a Ge thin film on an unsupported Si membrane causes large local distortions beneath the self-organized 50-nm-wide hut clusters. The huts also produce an overall, much smaller, curvature of the membrane that can be understood as an average response to a random distribution of huts. With appropriate growth conditions, even SOI template layers constrained by the buried oxide layer can be distorted by $\mathrm{Ge}$ huts. ${ }^{8,9}$ In this letter, we present a conceptually simpler approach: to fabricate Ge huts on freestanding template layers. These layers can be deformed without requiring the

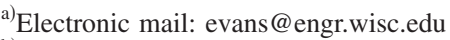

${ }^{b}$ Present address: Materials Research Institute, Pennsylvania State University, University Park, PA 16801.
}

unusually high growth temperatures of $700{ }^{\circ} \mathrm{C}$ that must be used to cause $\mathrm{SiO}_{2}$ to flow beneath unreleased layers. ${ }^{8}$

Structures in which a region of a thin SOI template layer is supported along a single edge are shown in Fig. 1. The 100-nm-thick template layer of an SOI structure (obtained from SiGen, Inc., San Jose, CA) was thinned to $30 \mathrm{~nm}$ using a sacrificial thermal oxide that was subsequently removed with hydrofluoric acid (HF). The template layer was patterned into 5- $\mu \mathrm{m}$ square mesas using optical lithography and vertically etched to the handle wafer. The buried oxide beneath the mesas was partially removed using HF vapor ${ }^{10}$ to create a freestanding region $\sim 250 \mathrm{~nm}$ wide at the mesa edges that imitates a freestanding membrane. Ge huts were grown using molecular beam epitaxy to deposit $\mathrm{Ge}$ at $0.1 \mathrm{ML} \mathrm{min}^{-1}$ with the sample held at $550{ }^{\circ} \mathrm{C}$. The mean size of the huts was $50 \mathrm{~nm}$, measured using scanning electron microscopy following the deposition. The average separation of nearest-neighbor huts was $175 \mathrm{~nm}$.

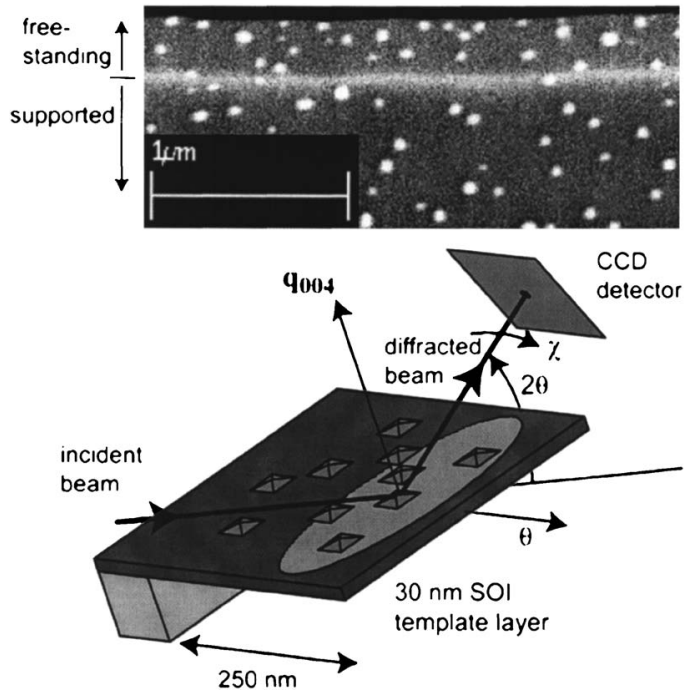

FIG. 1. (a) Top-view scanning electron microscope image of Ge hut clusters on a freestanding 30-nm-thick Si membrane. The SOI template layer is mechanically supported along the bottom edge of the image. (b) X-ray microdiffraction experiments allow structural studies using the $\mathrm{Si}(004)$ reflection from the template layer. The shaded ellipse represents the approximate footprint of the focused $\mathrm{x}$-ray beam. 


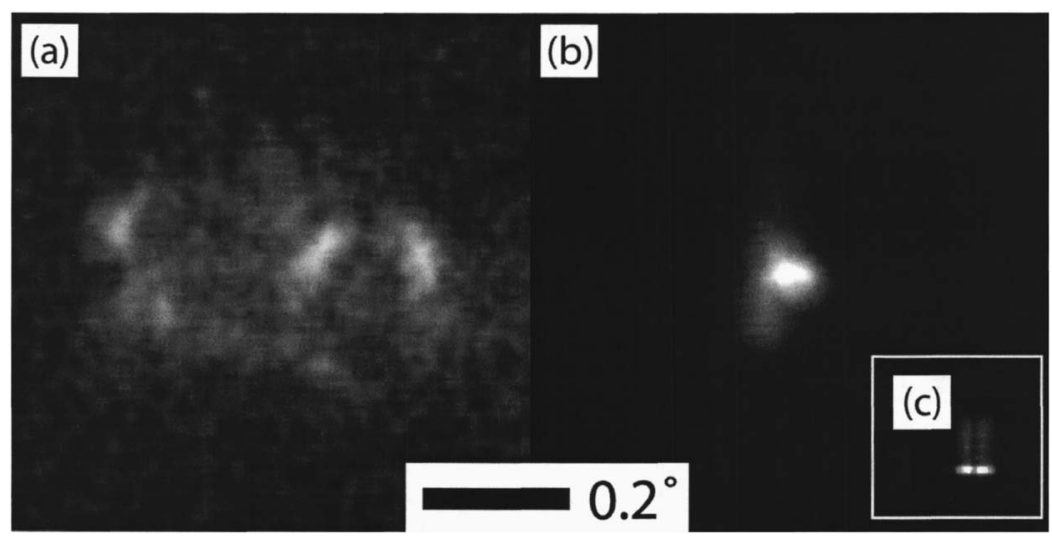

FIG. 2. Images of the $\mathrm{Si}(004) \mathrm{x}$-ray reflections of a freestanding $30 \mathrm{~nm}$ SOI template layer: (a) with Ge hut nanostressors, (b) without Ge. (c) The Si (004) reflection of the handle wafer shows a vertical streak caused by an artifact in the CCD camera used to acquire the diffraction patterns, and a shadow caused by the central stop that is part of the x-ray focusing apparatus (Ref. 11). The $\chi$ direction is horizontal in these images.

The strain induced by the huts into the Si template layer was determined using $\mathrm{X}$-ray microdiffraction at station 2ID-D of the Advanced Photon Source. ${ }^{11}$ The sample was aligned on a diffractometer with the incident beam positioned near the edge of the membrane. The $11.2 \mathrm{keV}$ x-rays from the undulator source were focused to a diameter less than $200 \mathrm{~nm}$ using a Fresnel zone plate. The elongated footprint of the beam was parallel to the edge so that the x-ray beam illuminated only the freestanding region (Fig. 1). A slight difference in the orientations of the handle wafer and template layer allowed their diffraction signatures to be studied simultaneously. Although present microdiffraction techniques lack the spatial resolution necessary to isolate a single Ge hut, unless they are grown several times farther apart, the spatial resolution is sufficient to study just a few adjacent huts with identical local conditions. A charge-coupled device (CCD) two-dimensional detector was used to measure the intensity of the diffracted beam on a plane spanned by two angles, $2 \theta$ and $\chi$. Here $2 \theta$ is the conventional angle between incident and diffracted beams and $\chi$ measures the lateral deflection of the diffracted x-ray beam. The bulk Si (004) reflection of the handle wafer (Fig. 2, inset) was obtained to determine the angular resolution of the system in the $\chi$ direction, which is limited to $0.05^{\circ}$ by the divergence introduced by the focusing optics.

The distortion of the Si template layer by the Ge huts broadens X-ray reflections from the membrane [Fig. 2(a)]. The diffracted beam is wider, in both the $\chi$ and $2 \theta$ directions, than the reflection from an area without Ge. The intensity of the diffracted beam as a function of $\chi$ has a width of $\Delta \chi$ $=0.5^{\circ}$. The diffraction pattern of an identically fabricated freestanding membrane that was shadowed during the $\mathrm{Ge}$ deposition [Fig. 2(b)], and which thus had no Ge huts stressing the membrane, has a full width at half maximum (FWHM) in the $\chi$ direction of $0.08^{\circ}$, sharper than the diffraction pattern of the region with Ge. This width is only slightly broader than the resolution set by the divergence of the x-ray beam.

Each diffraction pattern acquired by the CCD detector is a two-dimensional slice of the three-dimensional reciprocal space of the material illuminated by the focused $\mathrm{x}$-ray beam. The remaining dimension of reciprocal space can be explored by rotating the sample around an axis $\theta$ in the plane of the sample surface. By acquiring a diffraction pattern at each sample orientation we obtained the intensity distribution throughout the full volume of reciprocal space surrounding the (004) reflection. The angular widths in the $\theta$ direction of the template layer $\mathrm{Si}(004)$ x-ray reflections are approxiDownloaded 03 Mar 2007 to 128.104.198.190. Redistribution subject mately equal to the widths in the $\chi$ direction.

The broad diffraction pattern of the template layer in the region with Ge huts arises from the distortion of the Si (004) lattice planes by the Si-Ge lattice mismatch. This interpretation is bolstered by the equal widths of the diffraction pattern in the $\chi$ and $\theta$ directions, both of which correspond to a distribution of the Bragg condition over a wide range of sample orientations. One can obtain some insight into the deformation of the template layer by the huts with the assumption that the $\mathrm{Si}(004)$ planes are curved in a region approximately the size of one hut. The radius of curvature under the hut is then the hut size divided by the angular width of the diffraction spot. Using the diffraction pattern shown in Fig. 2, the radius of curvature beneath a $w$ $=50 \mathrm{~nm}$ hut would be $w / \Delta \chi \approx 6 \mu \mathrm{m}$. Membranes still supported by $\mathrm{SiO}_{2}$ served as control samples representing the response of rigid layers to the Ge huts. Huts did not detectably broaden $\mathrm{x}$-ray reflections from these areas.

The curvature found in this way can be compared to predictions of the mechanical response of the substrate to a localized stress. Continuum finite-element calculations and atomistic molecular dynamics models have been used to understand the mechanical response of a crystal to quantum dot structures $^{2}$ and, in particular, to find the strain in the Si substrate and cap layers near Ge huts. ${ }^{12,13}$ These calculations are most useful in describing the response of a thick substrate or a constrained template layer. In unsupported membranes, however, it is largely the average bending of the template layer by the island, analogous to large-scale substrate curvature, which results in the misorientation of the lattice planes in the template layer and broadens the x-ray reflections.

In a continuum-mechanics approximation, the curvature induced in a thick substrate by a thin blanket film is given by the Stoney equation, $\kappa_{s t}=6 m \varepsilon_{m} h_{f} / h_{s}^{2}$. Here $\varepsilon_{m}=4.2 \%$ is the mismatch between the lattice constants of Si and Ge, and $h_{f}$ and $h_{s}$ are the thicknesses of the film and substrate. The ratio of biaxial elastic constants is $m=0.79$ for Ge on $\mathrm{Si}^{14}$ Based on geometrical considerations, a 50-nm-wide (105) faceted hut is approximately $5 \mathrm{~nm}$ tall at its peak and has a mean thickness of $1.6 \mathrm{~nm}$. A naïve use of the Stoney equation with the thickness of the template layer $\left(h_{s} \approx 30 \mathrm{~nm}\right)$ and average height of the hut gives a radius of curvature of $2.8 \mu \mathrm{m}$. The Stoney equation can be corrected for the finite thickness of the substrate, which results in an increase in the predicted radius of curvature by $11 \%{ }^{3}$ A further correction for the shape and finite lateral size of the hut results in a decrease of $2 \%$ in the radius of curvature predicted for huts on 30 -nm-thick membranes. ${ }^{8}$ A likely origin of the difference to AIP license or copyright, see http://apl.aip.org/apl/copyright.jsp 


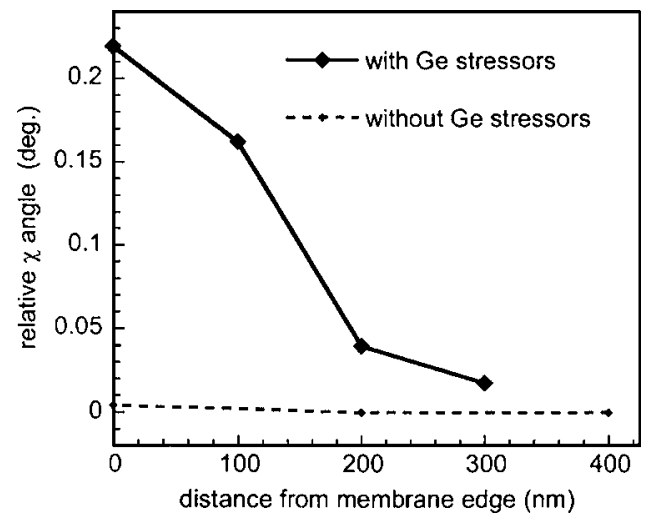

FIG. 3. Shift in the $\chi$ angle of the $\mathrm{Si}(004)$ reflection as a function of the distance of the $\mathrm{x}$-ray beam from the edge of the membrane. Overall curvature of the freestanding region induced by Ge huts causes the $\chi$ angle of the diffraction spot to vary.

between the radius of curvature predicted mechanically and the much larger experimentally observed value is the partial oxidation of the Ge huts under ambient conditions.

The template layer also responds to the huts with an overall downward bending towards the handle wafer. In our scattering geometry this bending is in the angular direction corresponding to the $\chi$ direction of the $\mathrm{Si}(004)$ diffraction patterns. The overall curvature of the membrane layer shifts the $\chi$ position of the center of the diffraction pattern, which depends on the position of the beam. The center of mass in $\chi$ of the diffracted beam shifts in $\chi$ by $0.2^{\circ}$ over the $250 \mathrm{~nm}$ width of the freestanding region (Fig. 3). This shift corresponds to an overall radius of curvature of $72 \mu \mathrm{m}$. On an unconstrained Si membrane, on which Ge huts are the only source of stress, the overall radius of curvature should be given by $\Delta x / \Delta \chi$, where $\Delta \chi$ is the angle the template layer is rotated by each hut and $\Delta x$ is the mean separation of huts. The overall radius of curvature predicted in this way is $\sim 20 \mu \mathrm{m}$, somewhat less than what is observed. This argument ignores the role of the $3 \mathrm{ML}$ wetting layer, which would produce an overall radius of curvature of $12 \mu \mathrm{m}$, even without huts. ${ }^{3}$ Oxidation of the Ge wetting layer and the partial oxidation of the huts may be responsible for the larger-than-expected local and extended radii of curvature observed experimentally.

Developing strain using individual Ge hut clusters complements two existing schemes for manipulating strain in thin silicon layers. Conventionally, strained Si layers have either been fabricated using compositionally graded SiGe alloy thin films that strain a subsequent epitaxially grown $\mathrm{Si}$ layer ${ }^{15}$ or using the strain-partitioning compliant-substrate approach. ${ }^{1,16}$ The elastic strain sharing approach, in particular, creates the potential for a new generation of high-speed $\mathrm{Si}$ electronic devices because relaxation occurs without dislocations. ${ }^{17}$ Even with our relatively thick $h_{s}=30 \mathrm{~nm}$ template layers, the difference in strain between the top and bottom of the membrane due to the bending induced by the hut nanostressors reaches $h_{s} / R=(30 \mathrm{~nm} / 6 \mu \mathrm{m})=0.5 \%$. The strain at the top surface of the template layer should reach half of this value, approximately $0.25 \%$. The Stoney equation predicts that the strain should increase as $1 / h_{s}$ and that a strain difference of $1 \%$ or more can thus be reached by using only slightly thinner template layers. In estimating the extreme limits of this approach, we note that SOI template layers have already been thinned reproducibly to the singleDownloaded 03 Mar 2007 to 128.104.198.190. Redistribution subject

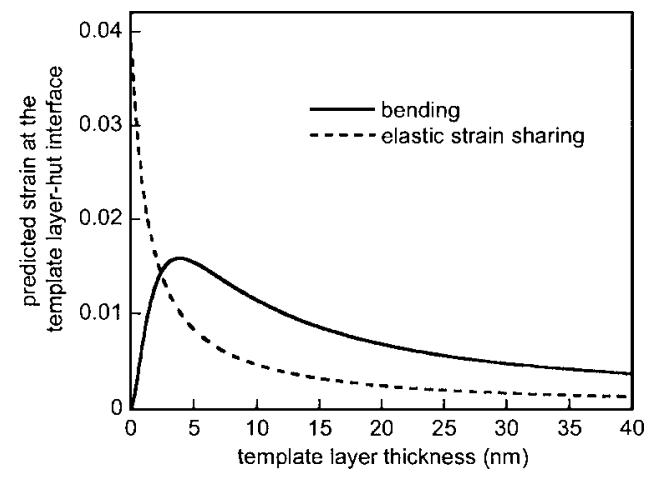

FIG. 4. Predictions of the elastic strain at the interface between a Ge hut and a freestanding Si template layer. The strain due to the curvature of the template layer exceeds the strain due to strain sharing for all template layer thicknesses above $2.4 \mathrm{~nm}$.

nanometer scale and can be processed to produce thin membranes. ${ }^{18}$ At these nanometer thicknesses, corrections for huts on thin SOI substrates are necessary. ${ }^{3,8}$ In Fig. 4, $\frac{1}{2} h_{s} / R$ is estimated using Ref. 9 and compared to the strain predicted by elastic strain sharing. ${ }^{1}$ For all template layer thicknesses greater than $2.4 \mathrm{~nm}$, the predicted strain at the template layer-hut interface due to curvature exceeds the strain developed by elastic strain sharing, reaching a maximum of $1.6 \%$ on 4 -nm-thick template layers.

This work was supported by the National Science Foundation through the University of Wisconsin Materials Research Science and Engineering Center, Grant No. DMR0079983. Use of the Advanced Photon Source was supported by the U.S. Department of Energy, Office of Science, Office of Basic Energy Sciences, under Contract No. W-31-109Eng-38.

${ }^{1}$ P. M. Mooney, G. M. Cohen, J. O. Chu, and C. E. Murray, Appl. Phys. Lett. 84, 1093 (2004).

${ }^{2}$ J. Stangl, V. Holy, and G. Bauer, Rev. Mod. Phys. 76, 725 (2004).

${ }^{3}$ L. B. Freund, J. A. Floro, and E. Chason, Appl. Phys. Lett. 74, 1987 (1999).

${ }^{4}$ R. E. Martinez, W. M. Augustyniak, and J. A. Golovchenko, Phys. Rev. Lett. 64, 1035 (1990).

${ }^{5}$ A. J. Schell-Sorokin and R. M. Tromp, Phys. Rev. Lett. 64, 1039 (1990).

${ }^{6}$ J. A. Floro, E. Chason, R. D. Twesten, R. Q. Hwang, and L. B. Freund, Phys. Rev. Lett. 79, 3946 (1997).

${ }^{7}$ Y.-W. Mo, D. E. Savage, B. S. Swartzentruber, and M. G. Lagally, Phys. Rev. Lett. 65, 1020 (1990).

${ }^{8}$ F. Liu, P. Rugheimer, E. Mateeva, D. E. Savage, and M. G. Lagally, Nature (London) 416, 498 (2002).

${ }^{9}$ F. Liu, M. Huang, P. P. Rugheimer, D. E. Savage, and M. G. Lagally, Phys. Rev. Lett. 89, 136101 (2002).

${ }^{10}$ Y. Fukuta, H. Fujita, and H. Toshiyoshi, Jpn. J. Appl. Phys., Part 1 42, 3690 (2003).

${ }^{11}$ Z. Cai, B. Lai, Y. Xiao, and S. Xu, J. Phys. IV 104, 17 (2003).

${ }^{12}$ P. Sutter, E. Sutter, P. P. Rugheimer, and M. G. Lagally, Surf. Sci. 532535, 789 (2003).

${ }^{13}$ P. Raiteri, L. Migli, F. Valentionotti, and M. Celio, Appl. Phys. Lett. 80, 3736 (2002).

${ }^{14}$ W. A. Brantley, J. Appl. Phys. 44, 534 (1973).

${ }^{15}$ M. L. Lee, A. J. Pitera, and E. A. Fitzgerald, J. Vac. Sci. Technol. B 22, 158 (2004)

${ }^{16}$ Y. H. Lo, Appl. Phys. Lett. 59, 2311 (1991).

${ }^{17}$ K. Rim, K. Chan, L. Shi, D. Boyd, J. Ott, N. Klymko, F. Cardone, L. Tai, S. Koester, M. Cobb, D. Canaperi, B. To, E. Duch, I. Babich, R. Carruthers, P. Saunders, G. Walker, Y. Zhang, M. Steen, and M. Ieong, IEEE International Electron Devices Meeting, 8-10 Dec. 2003, IEDM '03 Technical Digest, pp. 3.1.1-3.1.4.

${ }^{18}$ B. Legrand, V. Agache, J. P. Nys, V. Senez, and D. Stievenard, Appl. Phys. Lett. 76, 3271 (2000). 